\title{
A strategy for identifying serodiagnostically relevant antigens of Leishmania or other pathogens in genetic libraries
}

\author{
Márcia C.A. Teixeira ${ }^{\mathrm{a}, \mathrm{b}}$, Geraldo G.S. Oliveira ${ }^{\mathrm{a}}$, Marco A. Silvany ${ }^{\mathrm{a}, \mathrm{b}}$, \\ Neuza M. Alcântara-Neves ${ }^{\mathrm{a}, \mathrm{c}}$, Milena B.P. Soares ${ }^{\mathrm{a}}$, Ricardo Ribeiro-dos-Santos ${ }^{\mathrm{a}}$, \\ Selma M.B. Jerônimo ${ }^{\mathrm{d}}$, Carlos H. Costa ${ }^{\mathrm{e}}$, Washington L.C. dos-Santos ${ }^{\mathrm{a}}$, \\ Daniel Eichinger ${ }^{\mathrm{f}}$, Lain Pontes-de-Carvalho ${ }^{\mathrm{a}, *}$ \\ ${ }^{a}$ Centro de Pesquisas Gonçalo Moniz, Fundação Oswaldo Cruz, Rua Valdemar Falcão 121, 40295-001, Salvador, Brazil \\ ${ }^{\mathrm{b}}$ Faculdade de Tecnologia e Ciências - FTC, Salvador, Brazil \\ ${ }^{\mathrm{c}}$ Universidade Federal da Bahia, Salvador, Brazil \\ ${ }^{\mathrm{d}}$ Universidade Federal do Rio Grande do Norte, Natal, Brazil \\ ${ }^{\mathrm{e}}$ Universidade Federal do Piauí, Teresina, Brazil \\ ${ }^{\mathrm{f}}$ New York University Medical Center, New York, NY, USA
}

Received 2 October 2005; revised 3 January 2006; accepted 17 January 2006

\begin{abstract}
Different individuals, when infected with the same parasite, rarely produce antibodies against the same set of antigens. Indeed, unless a particular antigen happens to be recognized by antibodies in all individuals, the use of a single antigen in the serodiagnosis of parasitic diseases leads, invariably, to false-negative results. A straightforward method for pin-pointing, in genetic libraries, the precise antigens that would increase serodiagnostic assay sensitivities is presented. The method is based on the utilization of sera that produced false-negative results against previously available antigens. Employing this false-negative serum-selection methodology for the identification of new Leishmania infantum recombinant antigens (rAgs), the sensitivity of a dipstick assay for anti-Leishmania antibodies in a panel of sera from patients with visceral leishmaniasis was increased from $83.3 \%$ to $98.1 \%$, without affecting its specificity, by the inclusion of a fifth and a sixth $L$. infantum rAg.
\end{abstract}

(C) 2006 The International Association for Biologicals. Published by Elsevier Ltd. All rights reserved.

Keywords: Recombinant antigen; cDNA library; Serodiagnosis; Immunoassay sensitivity; Leishmania infantum; Visceral leishmaniasis

\section{Introduction}

Obtaining several milligrams of a pure protein from a complex microorganism was not an easy task a few years ago. The recombinant DNA technology, however, is changing this situation: many homogeneously pure microbial antigens can now be obtained in large amounts with relative ease. Serodiagnostic assays of several infectious diseases, therefore, increasingly employ recombinant [1-4] rather than native antigens. A

\footnotetext{
* Corresponding author. Tel.: +55 71 3560129; fax: +55 713562155 .

E-mail address: lain@cpqgm.fiocruz.br (L. Pontes-de-Carvalho).
}

major obstacle, however, limits the use of a single antigen in the serodiagnosis: the specificities of the immune responses against complex microorganisms vary in different individuals, and, to the authors' best knowledge, no single antigen is recognized by antibodies in all of them. For instance, even when relatively few sera from Leishmania infantum- $(n=9)$ or Trypanosoma cruzi-infected $(n=8)$ human beings, or from rabbits immunized with a mixture of keyhole limpet hemocyanin and Mycobacterium tuberculosis antigens in mineral oil-saline emulsion $(n=7)$, were analysed by Western blot against L. infantum, T. cruzi or cross-reactive Trypanosoma brucei lysates, respectively, no single antigenic band was 
clearly recognized by all tested sera (Fig. 1; unpublished data). The solution for this problem, however, may be the employment of more than one antigen in a serodiagnostic assay: this would maximize the chances of detecting antibodies in all positive sera.

To improve a serodiagnostic recombinant antigen (rAg) panel, it would be desirable to pin-point, in genetic libraries, precisely the phages encoding the rAgs that might increase the assay sensitivity, ignoring those phages encoding already available antigens. This may be a difficult task-the best represented (not necessarily the most immunogenic, i.e., serodiagnosis-relevant) antigens in a library, after been easily
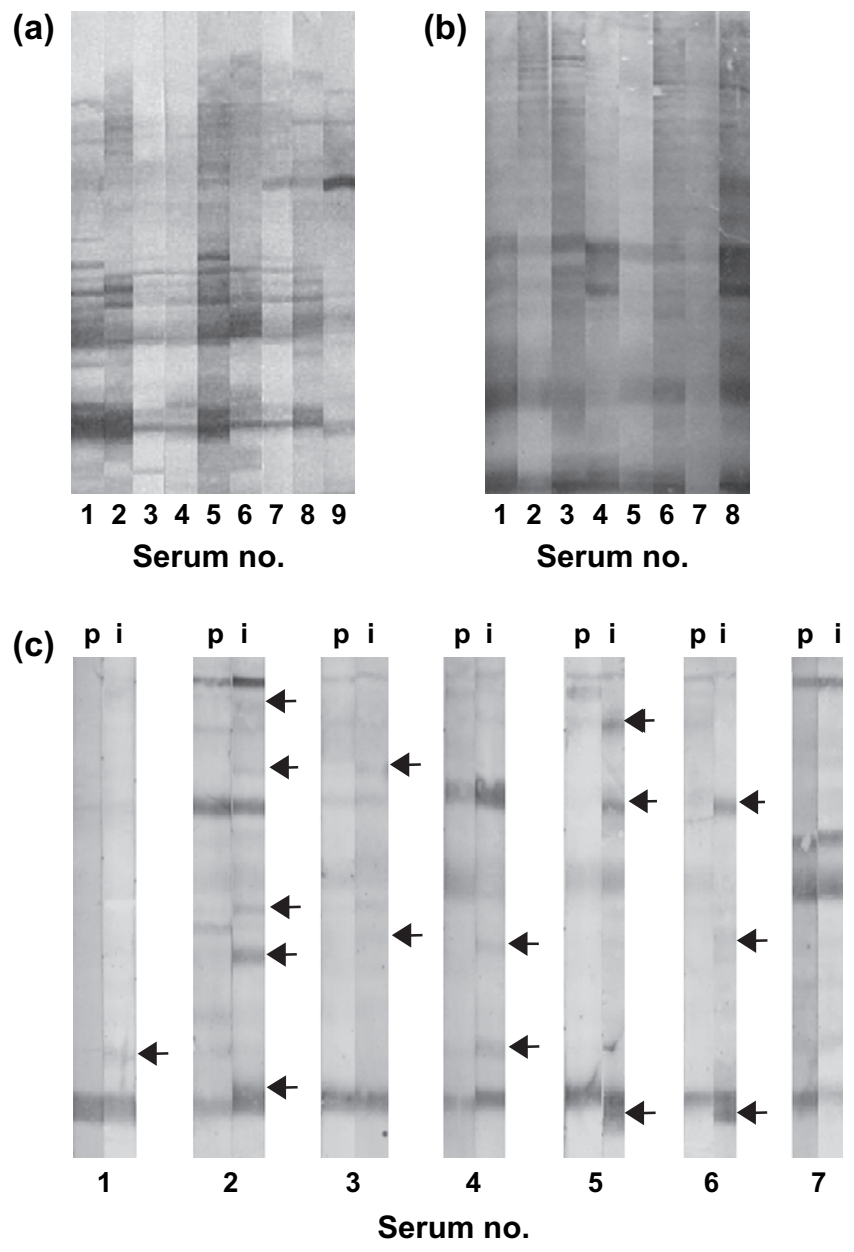

Fig. 1. Variability of immune responses in parasite-infected human beings or in animals immunized with complex antigenic mixtures. Sera from human beings naturally infected by Leishmania chagasi (a) or by Trypanosoma cruzi (b), and from New Zealand rabbits before and after immunization with a mixture of Mycobacterium tuberculosis and keyhole limpet hemocyanin (c), were used in Western blot assays against L. chagasi, T. cruzi and Trypanosoma brucei lysates, respectively. Each column corresponds to the reaction pattern of an individual serum. Arrows indicate antigens that were not recognized by antibodies in pre-immune serum. Note (i) how, although some antigens reacted strongly with antibodies from most human sera, none reacted with all sera (a and b), and (ii) the great variability of antibody specificities among individual rabbits, both in terms of "natural" (pre-immune) antibodies and of cross-reactive, post-immunization antibodies (c). $\mathrm{p}=$ pre-immune serum; $i=$ immune serum picked up, leave behind a high "background" of undesirable phages encoding them. This would obstruct the identification of the relatively small number of phages encoding the desired new antigens. A direct way for obtaining these relatively rare rAgs is to use, as a screening tool, exactly the sera previously producing false-negative results: these sera do not, by definition, recognize the already available antigens. This false-negative serum-selection strategy was employed as described below, in two quick runs, to identify two different $L$. infantum rAgs from a cDNA library. These rAgs, due to the very process through which they were obtained, are recognized by previously false-negative sera, and are therefore promising candidates to increase the sensitivity of a serodiagnostic assay for zoonotic visceral leishmaniasis (VL), a Latin American/ Southern European disease caused by the L. infantum protozoan $[5,6]$.

\section{Materials and methods}

\subsection{Sera}

Serum samples from 54 VL patients, inhabiting an endemic area of the state of Rio Grande do Norte, in the dry, poor northeastern region of Brazil, were used. All patients had diagnoses confirmed by demonstration of the parasite in bonemarrow aspirates. Control sera were from 22 informed healthy volunteers without history of leishmaniases. All sera were prepared from blood collected for immunodiagnostic purposes and for the development of serodiagnostic assays, in accordance with institutional ethical guidelines (which include patients' informed consent).

\subsection{Recombinant antigens and antigen-coating of nitrocellulose paper}

Nitrocellulose paper was individually coated, as described below, with (i) Leishmania rAgs in Escherichia coli lysates; (ii) negative control E. coli lysate; and (iii) positive control Leishmania lysate.

L. infantum amastigotes were obtained from the spleens of hamsters infected with $10^{8}$ metacyclic promastigotes and purified by centrifugation on a Percoll solution gradient [7]. The RNA isolated from purified amastigotes was used to construct a cDNA library in lambda ZAP bacteriophage (Stratagene, La Jolla, CA, USA) in accordance with manufacturer's instructions, in one of our laboratories. The library was screened with antibodies from pools of sera from (a) three dogs or (b) five human beings, all from VL endemic areas and with Leishmania amastigotes isolated from spleen or bone-marrow aspirates. Canine blood was collected by a veterinarian, in accordance with institutional ethical guidelines. Bacteriophages were isolated from the antibody-reacting plaques and incubated with $E$. coli agar cultures in Petri dishes, in the presence of isopropyl- $\beta$-D-thiogalactoside (IPTG), for the expression of the encoded recombinant proteins. The amount of added bacteriophages was that previously determined, by titration, to cause confluent lytic plaques on the bacterium layer 
after a 16-h incubation period at $37{ }^{\circ} \mathrm{C}$. After a 3-h incubation of the bacteriophage-bacterium mixture, nitrocellulose paper disks were juxtaposed onto the agar surface and incubated for $16 \mathrm{~h}$ at $37{ }^{\circ} \mathrm{C}$, in order to become coated with the expressed recombinant proteins. As negative control of solid-phase antigen, nitrocellulose disks were coated with non-specific protein lysates from agar plates in which $E$. coli was infected with non-recombinant lambda ZAP bacteriophages. L. infantum promastigotes, obtained from stationary-phase cultures in Schneider's insect cell medium containing $10 \%$ foetal bovine serum [8], were lysed by sonication at $4{ }^{\circ} \mathrm{C}$ (as a source of crude Leishmania antigen) and used to coat nitrocellulose paper at a concentration of $20 \mu \mathrm{g} \mathrm{ml}^{-1}$ of $0.15 \mathrm{M}$ phosphatebuffered saline, $\mathrm{pH} 7.2$ (PBS), during a 16-h incubation at $4{ }^{\circ} \mathrm{C}$. Possibly remaining protein-binding sites on recombinant and control antigen-coated nitrocellulose papers were blocked by incubation with $5 \%(\mathrm{w} / \mathrm{v})$ dry skimmed milk in PBS for at least $1 \mathrm{~h}$ at room temperature. Small $(8 \times 2 \mathrm{~mm})$ rectangular pieces were cut from the different antigen-coated nitrocellulose papers and transversally glued onto the same rigid plastic strip $(8 \times 700 \mathrm{~mm}$ dipstick), so as to form an array of several solid-phase antigens, disposed side by side, to be simultaneously tested in the dipstick assay. Selected phages were converted to plasmids by excision protocol (Stratagene) and had their inserted cDNA sequenced. Four different rAgs were used initially in the dipstick assay.

\subsection{Dipstick, ELISA and Western blot assays}

Sera were tested against the individual recombinant antigens by their incubation with the array of antigen-coated rectangular nitrocellulose paper pieces described above. Briefly, the antigen array was incubated with 1:400 dilutions of VL patients' or control sera. The binding of antibodies to the antigens was revealed by successive incubations with an antihuman $\operatorname{IgG~Fc}$-peroxidase conjugate (IgG-specific) and with a mixture of hydrogen peroxide and the diaminobenzidine chromogen (Sigma Chemical Co., St. Louis, MO, USA), as described elsewhere [9]. Western blots and ELISA were carried out with $L$. infantum lysates, as described previously $[10,11]$. A serum staining any of the $\mathrm{rAg}$-coated pieces of paper in the array was considered as producing a positive reaction.

\section{Results and discussion}

Twenty-two VL patients' sera were initially tested in a dipstick assay using four nitrocellulose paper pieces individually coated with the four rAgs that were obtained as described above (4-rAg dipstick assay). Two of these sera, however, despite reacting with the Leishmania lysate, did not react with any rAg (Fig. 2a). A pool of the two sera producing false-negative results in this 4-rAg assay was used to re-screen the cDNA library, leading to the identification of a fifth $\mathrm{rAg}$. This was used to set up a 5-rAg assay, which, in its turn, when tested against 32 previously untested VL patients' sera, led to the identification of four false-negative sera. A (a)

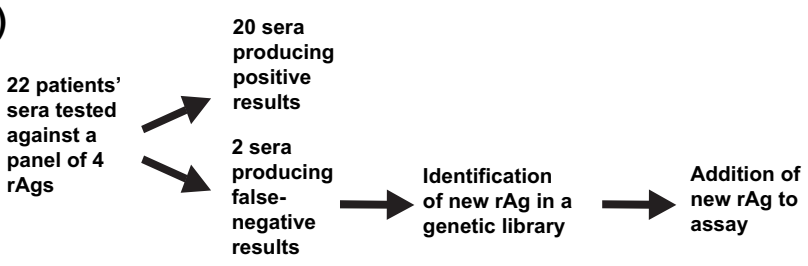

(b)

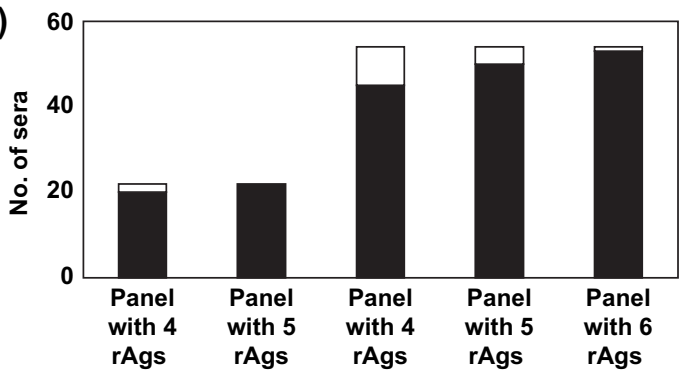

Fig. 2. Using sera producing false-negative results to obtain relevant recombinant antigens (rAgs). (a) Scheme of the procedure employed to improve an immunoassay for diagnosis of visceral leishmaniasis. (b) Number of sera from patients with visceral leishmaniasis recognizing at least one rAg in panels with increasing number of $L$. chagasi amastigote rAgs. The additional rAgs in the 5- and 6-antigen panels, in relation to the 4-antigen panel, were selected from a cDNA library as described in (a). Closed areas in the columns represent the number of sera producing positive results is an immunoenzymatic dipstick assay, in which each recombinant antigen was exposed separately to the sera, whereas the total height of the columns represent the total number of sera tested (22 or 54 sera).

pool of three of these new false-negative sera was then used for a third screening of the cDNA library, identifying a sixth antigen, which was also added to the dipstick assay. The reactivities of all 54 VL patients' and 22 control sera were finally assessed against each of the six rAgs in this last dipstick assay. Nine of the $54(16.7 \%)$ tested VL patients' sera did not react with any of the original four rAgs (Fig. 2b). A fifth rAg, obtained by means of screening a $L$. infantum cDNA library by a pool of two sera that did not recognize any of the four rAgs, was recognized by five of these nine initially falsenegative sera (including the two sera used in its selection). An immunoassay utilizing the five rAgs, therefore produced only four (7.4\%) false-negative results when tested against the 54 sera. A sixth rAg, obtained with the use of a pool of three of these four remaining false-negative sera, were used to set up a 6-rAg dipstick assay, which produced only one false-negative result (1.9\%) when tested against the 54 sera (Fig. 2b). This remaining false-negative serum did not react with $L$. infantum native antigens, either in an ELISA, a Western blot or in the dipstick assay.

Although, as mentioned above, only one (1.9\%) of the 54 sera failed to recognize at least one of the six rAgs, each of these rAgs, when individually analysed, were not recognized by $12(22.2 \%)$ to $37(68.5 \%)$ of the 54 sera. In addition, no rAg shared with another the reactivity against the same set of sera, demonstrating that the six rAgs differed antigenically among themselves. None of the 22 control sera reacted with any of the six rAgs in the dipstick assay (not shown). 
The false-negative serum-selection methodology has been employed, in two runs, as described herein, to expand a panel of $L$. infantum rAgs from four to six antigens, increasing from 83.3 to 98.1 the percentage of 54 VL patient's sera that reacted with at least one of the antigens in a dipstick assay. These six rAgs were not recognized by antibodies from 22 healthy individuals' sera in the dipstick assay, indicating that they are promising candidates for being included in serodiagnostic assays. The rAgs used in the work described herein should be tested against large numbers of sera from patients with LV, other clinical forms of leishmaniasis and other infectious and non-infectious diseases, and from healthy individuals, in order to allow the determination of the specificity and sensitivity of immunoassays employing them.

The sensitivity of a serological assay would of course reach $100 \%$ only if all infected or sick individuals made antibodies, something that would hardly happen in the first few days after an infection or in some immunodeficient individuals. In fact, antibodies against crude $L$. infantum antigens were not detected in one of the 54 studied VL patients' sera, making it impossible, whichever assay were used, to obtain an $100 \%$ positive record with this particular set of 54 sera.

The exposure of isolated rAgs in individual spots on the solid phase (rather than using an antigenic mixture), as performed in the work described herein, may have the effect of increasing the assay specificity, since it would allow the visualization of antibody reactions against individual rAgs. This might disclose possible infection- or disease-specific reaction patterns (as happens, for instance, for Western blots in HIV infection). The isolated application of rAgs to the solid phase has the additional advantage of increasing the density of each antigen. In the alternative case of utilizing an antigenic mixture, if any of the antigens in the mixture is poorly recognized by antibodies in a particular serum, it will "dilute out" the remaining antigens in the solid phase. This, complying with the association affinity constant equation [12], would obligatorily decrease the binding of antibodies to the solid phase and reduce the assay sensitivity.

Despite the simplicity and potential usefulness of the falsenegative serum-selection method described herein, saving both assay developer's time and expensive reagents, to the authors' best knowledge it has not been reported previously, and it may therefore have been unduly neglected as a quick process to pick up serodiagnostically relevant antigens in genetic libraries.

\section{Acknowledgments}

This work was financially supported by the Brazilian Ministry of Science and Technology (PRONEX, PADCT and $\mathrm{CNPq}$ ) and the State Government of Bahia, Brazil (CADCT/ FAPESB); D.E. was supported by US NIH grant AI44893. We thank Ms. Fabíola Nascimento for reviewing the English language of this manuscript.

\section{References}

[1] Flannery B, Costa D, Carvalho FP, Guerreiro H, Matsunaga J, Da Silva ED, et al. Evaluation of recombinant Leptospira antigen-based enzyme-linked immunosorbent assays for the serodiagnosis of leptospirosis. J Clin Microbiol 2001;39:3303-10.

[2] Da Silveira JF, Umezawa ES, Luquetti AO. Chagas disease: recombinant Trypanosoma cruzi antigens for serological diagnosis. Trends Parasitol 2001;17:286-91.

[3] Jiang J, Marienau KJ, May LA, Beecham 3rd HJ, Wilkinson R, Ching WM, et al. Laboratory diagnosis of two scrub typhus outbreaks at Camp Fuji, Japan in 2000 and 2001 by enzyme-linked immunosorbent assay, rapid flow assay, and Western blot assay using outer membrane 56kD recombinant proteins. Am J Trop Med Hyg 2003;69:60-6.

[4] Suh IB, Choi HK, Lee SW, Woo SK, Kang HY, Won YD, et al. Reactivity of sera from cases of Plasmodium vivax malaria towards three recombinant antigens based on the surface proteins of the parasite. Ann Trop Med Parasitol 2003;97:481-7.

[5] Lainson R. The American leishmaniases: some observations on their ecology and epidemiology. Trans R Soc Trop Med Hyg 1983;77:569-96.

[6] Fenech FF. Leishmaniasis in Malta and the Mediterranean basin. Ann Trop Med Parasitol 1997;91:747-53.

[7] Chang KP. Human cutaneous leishmania in a mouse macrophage line: propagation and isolation of intracellular parasites. Science 1980;209: 1240-2.

[8] Eperon S, McMahon-Pratt D. Extracellular cultivation and morphological characterization of amastigote-like forms of Leishmania panamensis and L. braziliensis. J Protozool 1989;36:502-10.

[9] Chu NM, Janckila AJ, Wallace JH, Yam LT. Assessment of a method for immunochemical detection of antigen on nitrocellulose membranes. J Histochem Cytochem 1989;37:257-63.

[10] Schenkman S, Pontes de Carvalho LC, Nussenzweig V. Trypanosoma cruzi trans-sialidase and neuraminidase activities can be mediated by the same enzymes. J Exp Med 1992;175:567-75.

[11] Paranhos-Silva M, Freitas LAR, Dos Santos WLC, Grimaldi Jr G, Pontes de Carvalho LC, Oliveira-dos-Santos AJ. A cross-sectional serodiagnostic survey of canine leishmaniasis due to Leishmania chagasi. Am J Trop Med Hyg 1996;55:39-44.

[12] Roitt IM, Delves PJ. Essential immunology. 10th ed. Oxford: Blackwell Science Ltd.; 2001. 